\title{
STRICTLY POSITIVE DEFINITE KERNELS ON COMPACT TWO-POINT HOMOGENEOUS SPACES
}

\author{
V. S. Barbosa And V. A. Menegatto
}

Abstract. We present a necessary and sufficient condition for the strict positive definiteness of a real, continuous, isotropic and positive definite kernel on a compact two-point homogeneous space. The characterization is achieved using special limit formulas for Jacobi polynomials and antipodal manifolds attached to points in the homogeneous spaces. The characterization recovers that one presented in D. Chen et al. (2003) in the case in which the space is a sphere of dimension at least 2, adds to that in Menegatto et al. (2006) in the case in which the space is the unit circle and that in Beatson and zu Castell (2011) in the case of a real projective space. As an application, we use the characterization to improve upon a recent result on the differentiability of positive definite kernels on the spaces.

Mathematics subject classification (2010): 22F30, 33C50, 33C55, 41A63, 42A82.

Keywords and phrases: Strict positive definiteness, isotropy, two-point homogeneous spaces, Jacobi polynomials, differentiability, addition formula.

\section{REFERENCES}

[1] R. Askey, Orthogonal polynomials and special functions, Society for Industrial and Applied Mathematics, Philadelphia, Pa., 1975.

[2] V. S. Barbosa And V. A. Menegatto, Differentiable positive definite functions on two-point homogeneous spaces, J. Math. Anal. Appl. 434 (2016), no. 1, 698-712.

[3] R. K. Beatson And W. ZU Castell, Scattered data interpolation of Radon data, Calcolo 48 (2011), no. 1, 5-19.

[4] S. Bochner, Hilbert distances and positive definite functions, Ann. of Math. 42 (1941), 647-656.

[5] B. Bordin, A. K. Kushpel, J. Levesley And S. A. Tozoni, Estimates of n-widths of Sobolev's classes on compact globally symmetric spaces of rank one, J. Funct. Anal. 202 (2003), no. 2, 307-326.

[6] G. BROWN AND FENG DAI, Approximation of smooth functions on compact two-point homogeneous spaces, J. Funct. Anal. 220 (2005), no. 2, 401-423.

[7] E. CARTAN, Sur certaines formes Riemanniennes remarquables des géométries à fondamental simple, (French) Ann. Sci. École Norm. Sup. (3) 44 (1927), 345-467.

[8] D. Chen, V. A. Menegatto and Xingping Sun, A necessary and sufficient condition for strictly positive definite functions on spheres, Proc. Amer. Math. Soc. 131 (2003), no. 9, 2733-2740.

[9] G. Everest, A. van Der Poorten, I. ShPARLins Ki AND T. WARD, Recurrence sequences, Mathematical Surveys and Monographs, 104. American Mathematical Society, Providence, RI, 2003.

[10] R. GAngolli, Positive definite kernels on homogeneous spaces and certain stochastic processes related to Lévy's Brownian motion of several parameters, Ann. Inst. H. Poincaré Sect. B (N.S.) 3 (1967), 121-226.

[11] E. GINÉ, The addition formula for the eigenfunctions of the Laplacian, Advances in Math. 18 (1975), no. $1,102-107$.

[12] S. Helgason, The Radon transform on Euclidean spaces, compact two-point homogeneous spaces and Grassmann manifolds, Acta Math. 113 (1965), 153-180.

[13] T. KOORNWINDER, The addition formula for Jacobi polynomials and spherical harmonics. Lie algebras: applications and computational methods (Conf., Drexel Univ., Philadelphia, Pa., 1972). SIAM J. Appl. Math. 25 (1973), 236-246. 
[14] A. Kushpel And S. A. Tozoni, Entropy and widths of multiplier operators on two-point homogeneous spaces, Constr. Approx. 35 (2012), no. 2, 137-180.

[15] V. A. Menegatto, C. P. Oliveira And A. P. Peron, Strictly positive definite kernels on subsets of the complex plane, Comput. Math. Appl. 51 (2006), no. 8, 1233-1250.

[16] T. NAGANO, Homogeneous sphere bundles and the isotropic Riemann manifolds, Nagoya Math. J. 15 (1959), 29-55.

[17] S. S. Platonov, On some problems in the theory of the approximation of functions on compact homogeneous manifolds, (Russian) Mat. Sb. 200 (2009), no. 6, 67-108; translation in Sb. Math. 200 (2009), no. 5-6, 845-885.

[18] I. J. Schoenberg, Positive definite functions on spheres, Duke Math. J. 9, (1942), 96-108.

[19] G. SzEGö, Orthogonal polynomials, Fourth edition. American Mathematical Society, Colloquium Publications, Vol. XXIII, American Mathematical Society, Providence, R.I., 1975.

[20] J. A. TiRAo, Antipodal manifolds in compact symmetric spaces of rank one, Proc. Amer. Math. Soc. 72 (1978), no. 1, 143-149.

[21] Yuan Xu And E. W. Cheney, Strictly positive definite functions on spheres, Proc. Amer. Math. Soc. 116 (1992), no. 4, 977-981.

[22] Hsien-Chung Wang, Two-point homogeneous spaces, Ann. Math. 55 (1952), no. 2, 177-191.

[23] J. ZIEGEL, Convolution roots and differentiability of isotropic positive definite functions on spheres, Proc. Amer. Math. Soc. 142 (2014), no. 6, 2063-2077. 